\title{
Morphological and functional changes in random donor platelets stored for seven days in platelet additive solution
}

\author{
Tulika Chandra, Ashish Gupta, Ashutosh Kumar, Sheeba Afreen
}

\begin{abstract}
Introduction: Platelets are small, anucleated cytoplasmic fragments that play an essential role in blood clotting and wound healing. Platelets are routinely stored in plasma for five days at $22^{\circ} \mathrm{C}$. Aim: Our objective was to assess platelet morphology and function in random donor platelet stored for seven days in platelet additive solution. Methods: The random donor platelet was prepared by PRP method. Platelet concentrates from 50 blood donor were stored in 100\% plasma $(n=24)$ and $20: 80 \quad(n=24)$ additive solution. The RDP were placed in incubator and agitator at $22^{\circ} \mathrm{C}$. Results: Out of total 50 samples, 48 samples were analyzed and two were discarded due to the bacterial contamination. On comparing the mean values of platelet count and MPV in both the groups with and without platelet additive solution, no significant difference was observed on day seven of storage period. A significant difference in PDW was observed on day seven $(p<0.001)$ in
\end{abstract}

${ }^{1}$ Tulika Chandra, ${ }^{1,2}$ Ashish Gupta, ${ }^{2}$ Ashutosh Kumar, 1,2Sheeba Afreen

Affiliations: ${ }^{1}$ Department of Transfusion Medicine and Blood Bank, 2Department of Pathology, Chhatrapati Shahuji Maharaj Medical University (Earlier King Georg's Medical University), Lucknow, Uttar Pradesh, India.

Corresponding Author: Dr. Tulika Chandra Assistant Prof. Department of Transfusion Medicine and Blood Bank Chhatrapati Shahuji Maharaj Medical University (Earlier King Georg's Medical University) Lucknow, Uttar Pradesh India Pin- 226003; Mo: +91-9415755536; Email: tulikachandra@rediffmail.com

Received: 12 October 2011

Accepted: 09 December 2011

Published: 31 December 2011 plasma. The mean values of $\mathrm{LDH}$ and $\mathrm{pH}$ showed no significant difference on day seven in both the groups with and without platelet additive solution. A significant difference was observed in the levels of glucose on day seven (p $<0.001$ ) in plasma. In platelet aggregation, a significant decrease was seen in plasma $(p<$ 0.001) on day seven at $22^{\circ} \mathrm{C}$. Conclusion: The platelet morphology and function was better maintained in additive solution on day seven as compared to platelets stored without additive solution.

Keywords: Platelet morphology, Platelet function, Random donor platelets, Platelet additive solution, Day

$* * * * * * * * *$

Chandra T, Gupta A, Kumar A, Afreen S. Morphological and functional changes in random donor platelets stored for seven days in platelet additive solution. International Journal of Blood Transfusion and Immunohematology 2011;1:20-25.

Article ID: 100005IJBTITC2011

$* * * * * * * * *$

doi:10.5348/ijbti-2011-5-OA-5

\section{INTRODUCTION}

Platelets participate in a number of physiological reactions which contribute to maintain thrombotic hemostasis in circulation. When activated by exposed subendothelium or by circulating agonists, platelets form aggregates which are incorporated into a platelet plug that prevents local hemorrhage $[1,2]$. Platelets are small, anucleated cytoplasmic fragments -that play an 
essential role in blood clotting and wound healing. There is increased demand for stored platelet concentrates (PCs) for therapeutic transfusions such as treatment of patients with disorders resulting in thrombocytopenia and for patients who become thrombocytopenic after chemotherapy or major invasive procedures, such as cardiac surgery. High platelet quality would be expected to result in improved clinical efficacy, determined by count increment, improved hemostasis, and lower risk for adverse reactions in recipients [3]. Increasing the storage time of platelet concentrates have always been a challenge in Transfusion medicine and many studies have been carried out with the aim of improving it [4-6]. Survival of platelets, like that of all other living systems, depends on the maintenance of a delicate biochemical balance between different substances including, in particular, glucose and hydrogen ions [7]. Platelets are routinely stored in plasma for five days at $22^{\circ} \mathrm{C}$. The biochemical, structural and functional changes that occur during platelet storage under blood bank conditions are collectively known as platelet storage lesion. These lesions may have an impact on platelet viability and hemostatic function. Platelet storage lesion (PSL) is associated with morphological changes and platelet activation followed by microvesciculation and loss of function, leading to transfusion failure [8]. Optimized synthetic media might help attenuate the platelet storage lesion, thereby facilitating extended storage. Platelet additive solution replaces $70-80 \%$ of the plasma in the original platelet unit, these formulations are predicted to have additional benefits: reduced allergic and transfusion reactions [9], decreased transfusion of unwanted antibodies (e.g. ABO, HLA) and increased plasma made for fractionation [10]. Various laboratory tests have been recommended to study platelet storage lesion ranging from most simple test such as $\mathrm{pH}$ to more complex tests of platelet function. Recently platelet indices such as platelet count (PLT), mean platelet volume (MPV), platelet distribution width (PDW), and platelet-large cell ratio (P-LCR) have been used as markers for the quality control of PCs, as these reflect storage-induces shape changes in platelets [11]. However, Beyan et al. [12], claimed that in condition where platelet function should have been assessed, platelet indices alone are inappropriate and further evaluation is necessary with different methods. Therefore, in the present study an attempt was made to analyze platelet morphology and functions in random donor platelets, stored for seven days in platelet additive solution.

\section{MATERIALS AND METHODS}

The study sample included 50 blood donors. Detailed medical history of donors was taken to exclude any infection and disease in the collected samples. Written consent was also taken from them prior to donation regarding their acceptability for the tests to be carried out for the transfusion transmitted diseases. All the blood units were screened for Hepatitis B Virus (Hepalisa, J Mitra \& Co. Pvt. Ltd., New Delhi, India) Hepatitis C Virus (HCV Microlisa, J Mitra \& Co. Pvt. Ltd., New Delhi, India), Human Immunodeficiency Virus 1 and 2 (Microlisa - HIV, J Mitra \& Co. Pvt. Ltd., New Delhi, India). Method used was Enzyme Linked Immunosorbent Assay (Elisa plate washer version 3 and Elisa plate reader version no. 1.300, Robonik Pvt. Ltd., Navi Mumbai, India). Syphilis was tested by Rapid Plasma Reagin (RPR) method (Span Diagnostic Ltd., Surat, India).

Random donor platelets preparation: The Random donor platelets were prepared by platelet rich plasma (PRP) method [13]. The whole blood (350 ml) was collected in anticoagulant Citrate Phosphate Dextrose Adenine (CPDA) triple blood bags (HL Hemopack, Hindustan Latex Ltd. Kerala, India). After a resting time of 30 minutes, the whole blood was centrifuged in a Cryofuge 600oi (Heraeus-Kendro, Hanau, Germany) at $1750 \mathrm{~g}$ for eight minutes at $22^{\circ} \mathrm{C}$ to obtain platelet rich plasma (PRP). The obtained PRP was again centrifuged at $3850 \mathrm{~g}$ for eight minutes under same experimental conditions. After the final centrifugation, the supernatant platelet poor plasma (PPP) was separated, and the residual pellet with the platelets was resuspended in a mean volume of $50 \pm 0.9$ $\mathrm{ml}$ of plasma. For storage of platelets in additive solution, $10-15 \mathrm{ml}$ of plasma was left with the platelet concentrates, and eight $\mathrm{ml}$ of additive solution was added to every two $\mathrm{ml}$ of platelet concentrate to give an expected final concentration of $80 \%$ additive solution and $20 \%$ plasma. The volume of additive solution and stored platelets had a mean volume of $50 \pm 1 \mathrm{ml}$. Platelet additive solution contains 5.26 gm sodium chloride, 5.02 gm sodium gluconate, 2.22 gm sodium acetate anhydrous, $0.373 \mathrm{gm}$ potassium chloride, $0.305 \mathrm{gm}$ magnesium chloride hexahydrate, $3.213 \mathrm{gm}$ sodium citrate [14]. Random donor platelet was placed in a platelet incubator (Remi Instruments Ltd., Mumbai, India) with continuous agitation at 70 cycles/minutes during storage at $22^{\circ} \mathrm{C}$.

In vitro platelet assay: Sampling was done on days 0,5 and 7 under sterile condition in biosafety cabinet grade II. Platelet count, mean platelet volume (MPV) and platelet distribution width (PDW) was done by automated cell counter (MS4, Blood cell counter, Anand Group, HD Consortium, India). Lactate dehydrogenase (LDH) determination was conducted on random donor platelets samples: random donor platelets $(1 \mathrm{ml})$ were centrifuged at $3000 \mathrm{~g}$ for five min. The supernatant was used to quantify the LDH by Semi Automated, Microlab 300 (Merck Specialties Pvt. Ltd., Goa, India). Glucose determination was done by centrifuging $1 \mathrm{ml}$ of random donor platelets in fluoride oxalate vial at $3000 \mathrm{~g}$ for five min. The supernatant was used to quantify the glucose by Erbachem 5 Plus analyzer (Erba Diagnostic Mannhein Gmbh, Mannhein, Germany). The $\mathrm{pH}$ of all samples was assessed immediately after sampling at a temperature of $24^{\circ} \mathrm{C}$ using the Compla pH meter (Composite Lab Line Pvt. Ltd, Lucknow, India). The platelet aggregation was 
determined by the optical method [15] using a flatbottom aggregometer (Chornolog-Corporation, Havertown, PA). The functional study of random donor platelets was performed by the platelet aggregation, using as an agonist $5 \mu \mathrm{M}$ adenosine di phosphate (ADP) (Amresco, Solon Ind. Pkwy. Solon, OH) at different storage periods. Aerobic culture was performed for all the samples on day o, day 5 and day 7 using direct plating and Robertson Cook medium (RCM) simultaneously. In direct plating, $25 \mu \mathrm{l}$ samples was streaked on nutrient agar plate and MacConkey agar and incubated at $37^{\circ} \mathrm{C}$ for 48 hours. If growth occured in the medium, further identification of bacterial species was done by using standard laboratory techniques [16] and if no growth occured then the sample was considered as sterile. In RCM, we placed a drop containing $25 \mu \mathrm{l}$ of sample on Robertson Cook medium and the plate was incubated for 72 hours at $37^{\circ} \mathrm{C}$. When turbidity was observed in the sample, it was further processed according to direct plating method. Further result of RCM method was matched with result of direct plating, this ensured whether our direct plating method was performed correctly or not.

Statistical Analysis: Data were reported as means \pm standard deviation (SD). The data was compared using paired t-test. The confidence limit was kept at $95 \%$, hence a $\mathrm{p}<0.05$ was considered to be statistically significant.

\section{RESULTS}

Out of 50 samples, 48 samples were analyzed and two were discarded due to bacterial contamination. On comparing, the mean values of platelet count and MPV in the two groups (with and without platelet additive solution), no significant difference was observed on day 7 of storage period. Random donor platelet with platelet additive solution was the test group and its control group was random donor platelet without platelet additive solution. In each of the groups with and without platelet additive solution day 5 served as control while day 7 was taken as test group. There was no significant difference in PDW on day 7 in platelet additive solution. In contrast a significant difference in PDW was observed on day 7 ( $p<0.001)$ in plasma. The mean values of $\mathrm{LDH}$ and $\mathrm{pH}$ showed no significant difference on day 7 in both the groups with and without platelet additive solution. A significant difference was observed in the levels of glucose on day 5 and day 7 ( $p<0.001)$ in plasma. In contrast, no significant difference was observed in glucose levels on day 7 in platelet additive solution. In platelet aggregation a significant decrease was seen in plasma $(\mathrm{p}<0.001)$ and no significant difference in platelet additive solution on day 7 at $22^{\circ} \mathrm{C}$ (table 1).

\section{DISCUSSION}

The platelet storage lesion results from a complex process that is influenced by physical, chemical and metabolic factors related to platelet preparation and storage. Recently, changes in platelet indices during storage of platelet concentrates have found to be useful parameters to study platelet storage lesion $[17,18]$. Optimized synthetic storage media might help attenuate the platelet storage lesion, thereby facilitating extended storage. In an additive solution unit, the final medium contains 20-30\% donor plasma. This carried over plasma provides glucose for platelet metabolism. Platelet additive solution contains acetate, which serves as a second metabolic fuel. Acetate has the added benefit of acting as a buffer. Magnesium and potassium are present in platelet additive solution. These electrolytes inhibit platelet activation and aggregation, although how they work is unclear [10]. Potassium plays an important role in maintaining the platelet membrane potential,

Table 1: Comparison the parameters of random donor platelets stored for seven day at $22^{\circ} \mathrm{C}$ with and with out platelet additive solution.

\begin{tabular}{|c|c|c|c|c|c|c|c|}
\hline \multirow[t]{2}{*}{ S. No } & \multirow{2}{*}{ Parameter } & \multicolumn{3}{|c|}{ Platelet in platelet additive solution } & \multicolumn{3}{|c|}{ Platelet in plasma } \\
\hline & & o day & 5 day & 7 day & o day & 5 day & 7 day \\
\hline 1. & Platelet Count $\left(\mathrm{m} / \mathrm{mm}^{3}\right)$ & $246 \pm 34$ & $242 \pm 30$ & $240 \pm 30$ & $246 \pm 30$ & $243 \pm 30$ & $237 \pm 34$ \\
\hline 2. & MPV (fl) & $4.6 \pm 1$ & $4.8 \pm 1.3$ & $4.9 \pm 1.4$ & $4 \cdot 5 \pm 1$ & $4.9 \pm 1.4$ & $5 \cdot 4 \pm 1.3$ \\
\hline 3. & PDW & $5.0 \pm 1.5$ & $5.6 \pm 1.4$ & $6.0 \pm 1.4$ & $5.2 \pm 1.3$ & $5.8 \pm 1.4$ & $6.8 \pm 1.3^{*}$ \\
\hline $4 \cdot$ & $\mathrm{LDH}(\mathrm{U} / \mathrm{L})$ & $145 \pm 24$ & $148 \pm 22$ & $154 \pm 24$ & $139 \pm 23$ & $144 \pm 21$ & $147 \pm 22$ \\
\hline 5. & $\mathrm{pH}$ & $7.15 \pm 0.03$ & $7.13 \pm 0.04$ & $7.12 \pm 0.05$ & $7.14 \pm 0.05$ & $7.13 \pm 0.02$ & $7.09 \pm 0.04$ \\
\hline 6. & Glucose (mmol/L) & $12.9 \pm 2$ & $11.6 \pm 4$ & $8.9 \pm 4$ & $15 \cdot 4 \pm 2$ & $12.7 \pm 2$ & $6.0 \pm 4^{*}$ \\
\hline 7. & $\begin{array}{l}\text { Platelet aggregation \% } \\
(5 \mu \mathrm{M} \text { ADP) }\end{array}$ & $69 \pm 4$ & $62 \pm 4$ & $58 \pm 3$ & $72 \pm 4$ & $64 \pm 4$ & $40 \pm 3^{*}$ \\
\hline
\end{tabular}

$\mathrm{n}=48$ units of random donor platelets,

Value was mean $\pm \mathrm{SD} ;{ }^{*} \mathrm{p}<0.001$ compared to five day 
[19] and, when absent, potassium will leak rapidly from the platelet and needs to be recovered by energyrequiring potassium pumps. Furthermore, it has been shown that presence of external magnesium activates various potassium pumps [20]. Also, there is evidence that magnesium decreases the platelet activation [21] and influences the calcium influx into the platelets, thereby having an effect on the intracellular concentration of potassium [22].

Platelet can be prepared by random donor platelets, apheresis and by pooling of platelet units [13]. Random donor platelets were used in the present study. In the present study, platelet swirling was present in all the units at a temperature of $22^{\circ} \mathrm{C}$ in both the groups with and without platelet additive solution on day 7 . In the present study platelet count was maintained on day 7 at $22^{\circ} \mathrm{C}$ in both the groups with and without platelet additive solution. We observed that MPV was minimally increased in random donor platelet on day 7 in plasma and platelet additive solution.

Platelet distribution width (PDW) is a mixture of volume heterogeneity that measures platelet anisocytosis. PDW is an indication of variation in platelet size which can be a sign of active platelet release. In the present study we observed that PDW increased in random donor platelet on day 7 in both the groups with and without platelet additive solution. Lin et al. [23] investigated the effect of a platelet transfusion on such platelet parameters as the mean platelet volume (MPV), platelet distribution width (PDW), mean platelet component (MPC) and platelet component distribution width (PCDW) in transfused patients. There were no significant difference in any of the platelet parameters between the SDP $(n=21)$ and the pooled PC $(n=8)$. The MPC and PCDW of the SDP and the PC were significantly lower than those of the samples from the patients before transfusion. However, the PCDW of the samples from the patients after transfusion was significantly lower than that before transfusion.

In the present study it was observed that the $\mathrm{LDH}$ level slightly increased on day 7 in random donor platelets with and without platelet additive solution and observed that the level of $\mathrm{LDH}$ was maintained on day 7 at $22^{\circ} \mathrm{C}$. Among metabolic parameters $\mathrm{pH}$ showed a significant fall over seven days of storage. Fall in $\mathrm{pH}$ may affect the quality of final platelet product. The American Association of Blood Banks (AABB) [24] recommended that platelets with $\mathrm{pH}<6.2$ should not be used for transfusion, and in Europe the same recommendation applies to platelets with $\mathrm{pH}>7.4$ [25]. As per the Drug and Cosmetics Act of India [26], minimum $\mathrm{pH}$ should not be $<6.0$ at any given day of storage. If $\mathrm{pH}$ falls below 6.0 or rises above 7.4 , a disc to sphere transformation of the platelets takes place, resulting in marked loss of recovery in vivo upon transfusion [27]. In the present study we observed that $\mathrm{pH}$ value decreased slightly but was within acceptable range on day 7 at $22^{\circ} \mathrm{C}$ in both the groups with and without platelet additive solution of the storage period.
The three major points in the production and storage of platelet concentrates are essential to maintain good platelet quality. First, the activation of platelets during collection, preparation and storage of platelet concentrates should be prevented or at least reduced to a very low level. Second, the level of glycolytic activity, the anaerobic consumption of glucose and production of lactate should be kept to a minimum level. Third, at least some glucose should be present in the platelet concentrates throughout the whole storage period [28]. In the present study, the glucose level slightly decreased in random donor platelets with and without additive solution on day 7 at $22^{\circ} \mathrm{C}$. In the study by Amorini AM et al. [7], platelet concentrates were saved under gentle stirring at $22^{\circ} \mathrm{C}$ for a total period of eight days. Glucose $0.5 \%(\mathrm{w} / \mathrm{v})$ was added either at the beginning of storage (time o) or on the fifth day of storage. They investigated microbiological culture, $\mathrm{pH}, \mathrm{MPV}$ and metabolites of energy pathways on day $0,5,6,7$ and 8 of storage period. They observed that the addition of glucose $0.5 \%$ on day 5 did not produce significant differences in metabolites of energy pathways with respect to control platelets, whereas when the glucose was added at the beginning of storage (time o) there was a recovery of ATP, GTP and a decrease of energy catabolism, demonstrating a beneficial effect on energy metabolism. The improvements produced by addition of glucose at time o were confirmed by MPV and the $\mathrm{pH}$ level. Singh et al. [29] reported the platelet storage lesion in 40 units pooled platelet concentrates harvested by platelet rich plasma stored for seven day. They observed no significant changes in the indices without EDTA during storage, however, after EDTA incubation significant changes were noted in dPLT and dMPV. Metabolic parameters showed a significant decrease in $\mathrm{pH}$ and $\mathrm{pCO}_{2}$ concentration with increase in $\mathrm{pO}_{2}$ during storage ( $\mathrm{p}<0.05)$.

One of the most common methods of measuring platelet aggregation is called optical platelet aggregation. This technique, which is a high-complexity laboratory test, involves adding an aggregating agent (e.g. ADP, epinephrine, thrombin, arachidonic acid) to platelet-rich plasma, a turbid platelet-rich suspension derived from whole blood. The effect of the aggregating agent on the suspension's light transmittance is then measured to assess platelet aggregation [15]. In present study we observed that platelet aggregation were decreased in random donor platelets in both the groups with and without platelet additive solution on day 7 at $22^{\circ} \mathrm{C}$.

\section{CONCLUSION}

Our study infers that platelets stored in platelet additive solution show slight increase in MPV and PDW while a greater increase is seen in random donor platelets stored in plasma on $7^{\text {th }}$ day. The platelet morphology and function was better maintained in 
additive solution on day 7 as compared to platelets stored without additive solution.

$* * * * * * * * *$

\section{Author's Contribution}

Tulika Chandra - Substantial contributions to conception and design, Analysis and interpretation of data, Revising it critically for important intellectual content, Final approval of the version to be published Ashish Gupta - Analysis and interpretation of data, Drafting the article, Revising it critically for important intellectual content, Final approval of the version to be published

Ashutosh Kumar - Substantial contributions to conception and design, Drafting the article, Final approval of the version to be published

Sheeba Afreen - Analysis and interpretation of data, Drafting the article, Revising it critically for important intellectual content, Final approval of the version to be published

\section{Guarantor}

The corresponding author is the guarantor of submission.

\section{Conflict of interest}

Authors declare no conflict of interest.

\section{Source of Support}

Council of Science and technology, Uttar Pradesh, Lucknow

\section{Acknowledgement}

We are grateful to the Council of Science and Technology, Lucknow, Uttar Pradesh, for funding this work.

\section{Copyright}

(C) Tulika Chandra et al. 2011; This article is distributed under the terms of Creative Commons attribution 3.0 License which permits unrestricted use, distribution and reproduction in any means provided the original authors and original publisher are properly credited. (Please see www.ijbti.com/copyright-policy.php for more information.)

\section{REFERENCES}

1. Slichter S, Bolgiano D, Jones M, et al. Viability and function of 8-day stored apheresis platelets. Transfusion 2006;46:1763-9.

2. Thibault L, Beauesjour A, de Grandmont MJ, Lemieux R, Leblanc JF. Charecterization of blood componentes prepared from whole blood donations after a 24 hour hold with the platelet rich plasma method. Transfusion 2006;46:1292-9.

3. Maurer-Spurej E, Chipperfield K. Past and future approaches to assess the quality of platelet for transfusion. Transfus Med Rev 2007;21:295-6.
4. Badlou BA, Ijseldijk MJW, Smid WM. Prolonged platelet preservation by transient metabolic suppression. Transfusion 2005;45:214-2.

5. Heddle NM, Barty RL, Boye D. Evaluation of whole blood derived platelets stored as a pool for 7 days. Transfusion 2002;42:suppl S39-030G.

6. Pietersz RNI, Van der, Meer PF. Extended platelet shelf life. Transfus Apher Sci 2001;24:239-40.

7. Amorini AM, Tuttobene M, Lazzarino G, Denti G. Evaluation of biochemical parameters in platelet concentrates stored in glucose solution. Blood Transfus 2007;5:24-32.

8. Krishnan LK, Sulochana PV, Mathai J, John A. Morphological and ultrastructural changes of platelet concentrates stored in PVC bags. Indian J Med Res 1997;105:77-84.

9. de Wildt-Eggen J, Nauta S, Schrijver JG, van Marwijk Kooy M, Bins M, van Prooijen HC. Reactions and platelet increments after transfusion of platelet concentrates in plasma or an additive solution: a prospective, randomized study. Transfusion 2000;40:398-3.

10. Ringwald J, Zimmermann R, Eckstein R. The new generation of platelet additive solution for storage at 22 degree C: development and current experience. Transfus Med Rev 2006;20:158-64.

11. Singh H, Chaudhary R, Ray V. Platelet indices as quality markers of platelet concentrates during storage. Clin Lab Hemat 2003;25:307-10.

12. Beyan C, Kaptan K, Ifran A. Platelet count, mean platelet volume, platelet distribution width and platelet count do not correlate with optical platelet aggregation responces in healthy volunteers. Thrombo Thrombolysis 2006;22:161-4.

13. Saran RK. Transfusion medicine technical manual; (2nd ed): WHO; New Delhi, India 2003.

14. Zammit V. In vitro assessment of platelets stored for seven days in a platelet additive medium- a pilot study. Biomed Sci 2006;121:25-32.

15. Kandice KM. Importance of platelets and platelet response in acute coronary syndromes. Clin $\mathrm{J}$ Med 2009;76:S2-S7.

16. Forbes BA, Sahm DF, Weissfeld AS. Bailley and Scott's diagnostic microbiology 10th ed. St. Louis (CV): Mosby 1998.

17. Seghatchian J, Krailadsiri P. The platelet storage lesion. Transfus Med Rev 1997;11:130-44.

18. Seghatchian J, Alfonso ME, Vickers MV. Effect of $\mathrm{pH}$ and buffering condition on dMPV of three types of platelet concentrates. Transfus Sci 1997;8:109-3.

19. Ishikawa Y, Sasakawa S. Membrane potential of stored platelets and its effect on platelet functions. Thromb Res 1987;45:265-73.

20. Bara M, Guiet-Bara A, Durlach J. Regulation of sodium and potassium pathways by magnesium in cell membranes. Magnes Res 1993;6:167-77.

21. Gawaz M, Ott I, Reininger AJ, Neumann FJ. Effects of magnesium on platelet aggregation and adhesion. Magnesium modulates surface expression of glycoproteins on platelets in vitro and ex vivo. Thromb Haemost 1994;72:912-8.

22. Weis-Fogh US. The effect of citrate, calcium, and magnesium ions on the potassium movement across the human platelet membrane. Transfusion 1985;25:339-42.

23. Lim YA, Cho SR. Effect of Platelet Transfusion on the Platelet Parameters of Recipient. Korean J Hematol 2009;44:36-41. 
24. Sweeney J. Quality assurance and standards for red cells and platelets. Vox Sang 1998;74:201-5.

25. Recommendation No. R (95) 15. Guide to the preparation, use and quality assurance of blood components 7 th ed Strasbourg, France: European Council Press 2001:123.

26. Mallik V. Drug and Cosmetic Act 1940. 13th ed. Lucknow, India: EBC Publishing (P) Ltd; India 2001:144-51.

27. Murphy S, Gardner FH. Platelet storage at $22^{\circ} \mathrm{C}$ : Role of gas transport across plastic containers in maintenance of viability. Blood 1975;46:209-12.

28. Gulliksson $H$. Defining the optimal storage conditions for the long term storage of platelets. Transfus Med Rev 2003;17:209-15.

29. Singh H, Chaudhary R, Ray V. Evaluation of platelet storage lesions in platelet concentrates stored for seven days. Indian j Med Res 2003;118:243-6. 